MATHEMATICS OF COMPUTATION

Volume 81, Number 277, January 2012, Pages 605-622

S $0025-5718(2011) 02506-8$

Article electronically published on August 25, 2011

\title{
ON SEMIREGULAR PERMUTATIONS OF A FINITE SET
}

\author{
ALICE C. NIEMEYER, TOMASZ POPIEL, CHERYL E. PRAEGER, \\ AND ŞÜKRÜ YALÇINKAYA
}

\begin{abstract}
In this paper we establish upper and lower bounds for the proportion of permutations in symmetric groups which power up to semiregular permutations (permutations all of whose cycles have the same length). Provided that an integer $n$ has a divisor at most $d$, we show that the proportion of such elements in $S_{n}$ is at least $c n^{-1+1 / 2 d}$ for some constant $c$ depending only on $d$ whereas the proportion of semiregular elements in $S_{n}$ is less than $2 n^{-1}$.
\end{abstract}

\section{INTRODUCTION}

A permutation of a finite set $\Delta$ is called semiregular if all of its cycles have the same length, $\ell$ say, and $\ell>1$. In particular, semiregular elements have no fixed points in $\Delta$, that is to say, they are fixed point free. They play an important role in graph theory (see Section 2). However, whereas the statistical distribution of fixed point free permutations (also called derangements) in symmetric groups and other groups has been widely studied for several hundred years, that of semiregular and associated permutations is not so well understood. Indeed, the proportion of derangements of a set of size $n$ was obtained by P. R. de Montmort [17] in 1708. He proved that this proportion approaches $1 / e$ as $n$ approaches infinity. By contrast the proportion of semiregular permutations may be arbitrarily small. For example, if $|\Delta|=n$ is a (large) prime, then the semiregular permutations are just the $n$ cycles, and their proportion is $1 / n$. The aim of this paper is to study a sub-family of derangements from which semiregular permutations may be constructed, and in particular, to estimate the proportion of such permutations on a set of composite cardinality.

Let $b, n$ be natural numbers such that $b \geq 2$ and $b$ divides $n$, let $\Delta=\{1,2, \ldots, n\}$ and let $S_{n}$ denote the symmetric group consisting of all permutations of $\Delta$. Then each semiregular permutation of $\Delta$ of order $b$ is a product of $n / b$ cycles of length $b$. All such permutations are conjugate, and the proportion of them in $S_{n}$, for fixed $b$ and large $n$, (using the Stirling approximation for $(n / b) !)$ is

$$
\frac{1}{(n / b) ! b^{n / b}} \approx\left(\frac{e}{n}\right)^{n / b} \frac{1}{\sqrt{2 \pi n / b}}
$$

As $b$ ranges over all divisors of $n$, the largest contribution to the proportion of semiregular elements comes from the $n$-cycles (taking $b=n$ ), which have proportion

Received by the editor December 10, 2009 and, in revised form, November 16, 2010.

2010 Mathematics Subject Classification. Primary 20B05, 20 B25.

Key words and phrases. Semiregular permutations, derangements, generating function.

(C)2011 American Mathematical Society 
$1 / n$, and estimating quite crudely we see that the total number of semiregular elements is always less than $n^{-1}+4 n^{-3 / 2}$; see Lemma 2.1 .

The asymptotic estimate above implies that finding a semiregular element of given order $b$ by random selection in $S_{n}$ requires roughly $\Theta\left(n^{n / b+1 / 2}\right)$ selections of independent, uniformly distributed random elements 1 Such a search is infeasible when $n$ is large. We propose to construct semiregular elements of a given order $b$ by using random selection to find in $S_{n}$ an element some power of which is semiregular of order $b$. We call an element $g \in S_{n}$ pre-semiregular of order $b$ if $b$ divides the order $o(g)$ and $g^{o(g) / b}$ is semiregular. For example, if $b=p^{k}$ with $p$ a prime, then the pre-semiregular elements of order $b$ are the permutations with all cycle lengths having the same $p$-part $p^{m}$ for some $m \geq k$. The main result of this paper determines the proportion of these elements in a more general setting.

Theorem 1.1. Let $b, d, m$ be positive integers with $b, d \geq 2$ and let $t_{b, d}(m b)$ denote the proportion of elements of $S_{m b}$ such that all cycle lengths are divisible by $b$, and no cycle has length divisible by $d b$. Then, for any positive real number $\varepsilon \leq \frac{1}{2 d b}$, there exist positive functions $A:=A(b, d, \varepsilon)$ and $B:=B(b, d)$ such that

$$
\frac{A}{(m b)^{1-\frac{1}{b}+\frac{1}{b d}+\varepsilon}} \leq t_{b, d}(m b) \leq \frac{B}{(m b)^{1-\frac{1}{b}+\frac{1}{b d}}} .
$$

The purpose of this result is to estimate $t_{b, d}(m b)$ for symmetric groups with degree $m b$ large compared with $b$. However, the quantities $A, B$ are such that the result holds for all positive $m$. Setting $b=p^{k}$ and $d=p$, for $p$ a prime, we obtain the following corollary.

Corollary 1.2. Let $p$ be a prime, let $k$ and $m$ be positive integers, and let $0<\varepsilon \leq$ $\left(2 p^{k+1}\right)^{-1}$. Then the proportion of pre-semiregular permutations of order $p^{k}$ on a set of size $m p^{k}$ is at least

$$
t_{p^{k}, p}\left(m p^{k}\right) \geq A\left(p^{k}, p, \varepsilon\right) /\left(m p^{k}\right)^{1-\frac{p-1}{p^{k+1}}+\varepsilon},
$$

where $A\left(p^{k}, p, \varepsilon\right)$ is as in Theorem 1.1.

Remark 1.3. (a) Definitions of the quantities $A(b, d, \varepsilon)$ and $B(b, d)$ are given in Subsection 3.4.

(b) We emphasise that Corollary 1.2 provides asymptotic information for a fixed prime power $p^{k}$ and increasing $m$, but $A\left(p^{k}, p, \varepsilon\right)$ is such that the result holds for all $m$. In particular, the corollary applies where the set has prime size $p$. This is the case $k=m=1$. Here the pre-semiregular permutations are the same as the semiregular permutations, namely the $p$-cycles. Their proportion is $1 / p$. The lower bound given in Corollary 1.2 is $A(p, p, \varepsilon) / p^{1-\frac{1}{p}+\frac{1}{p^{2}}+\varepsilon}$, which at first sight seems too large. However (see Lemma 3.3 (ii)), $A(p, p, \varepsilon) \leq 1 / p^{\frac{1}{p}-\frac{1}{p^{2}}-\varepsilon} \leq 1 / p^{\frac{1}{p}-\frac{3}{2 p^{2}}}$ and hence the lower bound given by Corollary 1.2 is less than or equal to the true value $1 / p$.

We also derive a consequence of these results for the proportion of pre-semiregular permutations in $S_{n}$ when $n$ has some proper divisor of bounded size.

\footnotetext{
${ }^{1}$ For functions $f$ and $g$ on the natural numbers, we say that $f=\Omega(g)$ if there are constants $a>0$ and $N$ such that, for all $n \geq N, f(n) \geq a g(n)$. We also say that $f=O(g)$ if $g=\Omega(f)$, and $f=\Theta(g)$ if $f=\Omega(g)$ and $g=\Omega(f)$.
} 
Corollary 1.4. For $d \geq 4$, there exists a function $C(d)$ such that if an integer $n$ has a nontrivial divisor at most $d$, then the proportion of pre-semiregular elements in $S_{n}$ is at least $C(d) / n^{1-\frac{1}{2 d}}$.

We show in Lemma2.1 that the proportion of semiregular permutations in $S_{n}$ lies between $n^{-1}$ and $2 n^{-1}$. Thus, provided $n$ has a divisor of bounded size, Corollary 1.4 implies that the proportion of pre-semiregular elements is asymptotically greater than the proportion of semiregular elements. Moreover, if $n$ has a divisor of size at most $d$, then constructing a semiregular element by finding and 'powering up' a presemiregular element requires at most $O\left(n^{1-1 /(2 d)}\right)$ independent random selections, as compared to $\Theta(n)$ required to find a semiregular element purely by random selection.

It would be interesting to know the exact asymptotics, as $m$ increases, for the proportions $t_{b, d}(m b)$ estimated in Theorem 1.1. We obtain a closed form for the generating function for these quantities. Define $t_{b, d}(0)=1$ and

$$
T(x)=\sum_{m \geq 0} t_{b, d}(m b) x^{m} .
$$

Theorem 1.5. Let $b, d$ be integers with $b, d \geq 2$. Then, for $|x|<1$,

$$
T(x)=\frac{\left(1-x^{d}\right)^{1 / b d}}{(1-x)^{1 / b}} .
$$

Despite obtaining in Proposition 5.1 an expression for $t_{b, d}(m b)$ as a sum in terms of known quantities, we have been unable to find the exact asymptotic behaviour of the $t_{b, d}(m b)$. We suspect that the correct asymptotic is given by the upper bound in Theorem [1.1, see the discussion in Remark 3.4.

Organisation of the paper is as follows. In Section 2, we comment on the role of semiregular permutations in graph theory and prove a result about the proportion of semiregular elements in $S_{n}$. Proofs of Theorem 1.1 and its corollaries are given in Section 3. We prove Theorem 1.5 in Section 4 . We derive an expression for the $t_{b, d}(m b)$ in Section 5. A general discussion about the true asymptotic behaviour of the quantities $t_{b, d}(m b)$ is given in Remark 3.4, with computational evidence presented in the final Section 6 .

\section{Semiregular Permutations in Graph theory}

The existence of semiregular permutations is of importance in graph theory. Marušič [15] and Jordan [11] independently conjectured that, for every finite vertextransitive graph, there should exist a semiregular edge-preserving permutation of its vertex set. Such semiregular permutations give useful structural information about the graph, as well as assisting with graph construction and enumeration (see for example [13, Section 4]) and graph drawing (see for example [10]). The conjecture was proved for cubic graphs [16 in 1998 and locally-quasiprimitive graphs [9] in 2007 (and hence, in particular, for all arc-transitive graphs of prime valency). In fact for vertex-transitive cubic graphs it is known that the maximum size of a semiregular subgroup (containing only semiregular automorphisms) is unbounded as the number of vertices increases [4, 12]. In [2, Problem BCC15.12], Klin proposed a more general form of this conjecture, known as the Polycirculant Conjecture, stating that every transitive 2-closed permutation group contains a fixed point free element of prime order. (A permutation group $G$ is called 2-closed if any permutation which preserves 
the orbits of $G$ on ordered pairs belongs to $G$.) The transitive permutation groups having no nontrivial semiregular subgroups are called elusive and studied in [3, 7, 8]; see also 6 .

The following result gives a crude estimate for the proportion of semiregular permutations in $S_{n}$.

Lemma 2.1. Let $n \geq 2$ and $\operatorname{semi}(n)$ denote the proportion of semiregular elements in $S_{n}$. Then $n^{-1} \leq \operatorname{semi}(n)<n^{-1}+4 n^{-3 / 2}$, and $\operatorname{semi}(n)<2 n^{-1}$.

Proof. For each divisor $b$ of $n$ the proportion of semiregular elements in $S_{n}$ of order $b$ is $p_{b}=\frac{1}{(n / b) ! b^{n / b}}$ (see equation (10). Thus $\operatorname{semi}(n) \geq p_{n}=n^{-1}$. If $b<n$, then $p_{b} \leq p_{n / 2}=2 / n^{2}$ with equality only if $b=n / 2$. For each divisor $b$ of $n$, the integer $n / b$ also divides $n$, and at least one of $b, n / b$ is less than or equal to $\sqrt{n}$. Thus the number of divisors of $n$ is at most $2 \sqrt{n}$. It follows that

$$
\operatorname{semi}(n)=p_{n}+\sum_{b<n} p_{b} \leq n^{-1}+2 \sqrt{n} p_{n / 2}=n^{-1}+4 n^{-3 / 2}
$$

where the sum is over the proper divisors $b$ of $n$. Moreover, this inequality is never sharp since either the number of divisors is strictly less than $2 \sqrt{n}$, or some proper divisor $b$ is less than $n / 2$ so that $p_{b}<p_{n / 2}$. If $n \geq 16$, then this upper bound is at most $2 n^{-1}$. It is straightforward to check that $\operatorname{semi}(n)=\sum_{b} p_{b}<2 n^{-1}$ also holds for $n<16$.

\section{Estimation of the PRoportions $t_{b, d}(m b)$}

In this section we prove Theorem 1.1 To do this we first prove a recursion for the $t_{b, d}(m b)$. Recall that, for $b, d \geq 2$ and $m \geq 1, t_{b, d}(m b)$ is the proportion of elements of $S_{m b}$ such that all cycles have length a multiple of $b$, and no cycle has length divisible by $d b$. We set $t_{b, d}(0)=1$.

3.1. Recursion. Let $T_{b, d}(m b)$ be the set of elements $g \in S_{m b}$ such that all $g$ cycles in $\Delta=\{1,2, \ldots, m b\}$ have length a multiple of $b$, and no $g$-cycle has length divisible by $d b$. Note that $t_{b, d}(m b)=\left|T_{b, d}(m b)\right| /(m b)$ !, and by convention, we take $\left|T_{b, d}(0)\right|=1$. For $m \geq 1$, let

$$
N_{d}(m)=\{j \mid 1 \leq j \leq m, d \text { does not divide } j\} .
$$

For any $j \in N_{d}(m)$, let $T_{b, d}^{j}(m b)$ denote the set of all permutations in $T_{b, d}(m b)$ where 1 lies in a cycle of length $j b$. Then $T_{b, d}(m b)$ is the disjoint union of the $T_{b, d}^{j}(m b)$ for $j \in N_{d}(m)$.

Now we compute the size of $T_{b, d}^{j}(m b)$ for any $j \in N_{d}(m)$. We do this by constructing the set. For each element in $T_{b, d}^{j}(m b), 1$ is contained in a cycle of length $j b$, and the number of ways to choose the remaining $j b-1$ points of this cycle is $\left(\begin{array}{c}m b-1 \\ j b-1\end{array}\right)$. Moreover, for each choice of $j b$-subset, the number of ways to define a $j b$-cycle on it is $(j b-1)$ !, and the action on the remaining $(m-j) b$ points is one of 
$\left|T_{b, d}(m-j) b\right|$ possibilities. Therefore, we have

$$
\begin{aligned}
\left|T_{b, d}^{j}(m b)\right| & =\left(\begin{array}{c}
m b-1 \\
j b-1
\end{array}\right)(j b-1) !\left|T_{b, d}((m-j) b)\right| \\
& =\frac{(m b-1) !}{(m b-j b) !}\left|T_{b, d}((m-j) b)\right| \\
& =\frac{(m b-1) !}{(m b-j b) !}(m b-j b) ! t_{b, d}((m-j) b) \\
& =(m b-1) ! t_{b, d}((m-j) b) .
\end{aligned}
$$

Thus $\left|T_{b, d}(m b)\right|=(m b-1) ! \sum_{j \in N_{d}(m)} t_{b, d}((m-j) b)$, so

$$
m b t_{b, d}(m b)=\sum_{j \in N_{d}(m)} t_{b, d}((m-j) b) .
$$

In particular, for any $m<d$ we have

$$
m b t_{b, d}(m b)=\sum_{j=0}^{m-1} t_{b, d}(j b),
$$

and for $m=d$ we have $N_{d}(d)=N_{d}(d-1)$, so that

$$
d b t_{b, d}(d b)=\sum_{j=1}^{d-1} t_{b, d}(j b)=(d b-b+1) t_{b, d}((d-1) b)-1 .
$$

To derive a different form of the recursion for larger values of $m$, suppose that $m \geq d+1$. Note that $j \in N_{d}(m-d)$ if and only if $1 \leq j \leq m-d$ with $j \not \equiv 0$ $(\bmod d)$, which holds if and only if $d+1 \leq j+d \leq m$ with $j \not \equiv 0(\bmod d)$. Thus

$$
N_{d}(m)=\left\{j+d \mid j \in N_{d}(m-d)\right\} \cup\{1,2, \ldots, d-1\} .
$$

Considering equation (2) with $m-d$ in place of $m$, we see that

$$
m b t_{b, d}(m b)=(m-d) b t_{b, d}((m-d) b)+\sum_{j=1}^{d-1} t_{b, d}((m-j) b) .
$$

3.2. Small values of $m$. We provide expressions for $t_{b, d}(m b)$ for small values of $m$. These illustrate the variability of the quantities. Notice that the values are much smaller when $m$ is divisible by $d$.

Case $m=1$. $T_{b, d}(b)$ is the set of $b$-cycles in $S_{b}$, and so $t_{b, d}(b)=\frac{1}{b}$.

Case $m=2$. If $d>2$, then $T_{b, d}(2 b)$ consists of all elements $g \in S_{2 b}$ such that either $g$ is a $2 b$-cycle, or $g$ is a product of two $b$-cycles. On the other hand, if $d=2$, then $T_{b, d}(2 b)$ consists only of the products of two $b$-cycles. Thus

$$
t_{b, d}(2 b)= \begin{cases}\frac{1}{2 b^{2}} & \text { if } d=2, \\ \frac{1}{2 b}+\frac{1}{2 b^{2}} & \text { if } d \geq 3 .\end{cases}
$$

Case $m=3$. A similar computation gives

$$
t_{b, d}(3 b)= \begin{cases}\frac{1}{3 b}+\frac{1}{6 b^{3}} & \text { if } d=2, \\ \frac{1}{2 b^{2}}+\frac{1}{6 b^{3}} & \text { if } d=3 \\ \frac{1}{3 b}+\frac{1}{2 b^{2}}+\frac{1}{6 b^{3}} & \text { if } d \geq 4 .\end{cases}
$$


Case $m=4$. Similarly, we have

$$
t_{b, d}(4 b)= \begin{cases}\frac{1}{3 b^{2}}+\frac{1}{24 b^{4}} & \text { if } d=2, \\ \frac{1}{4 b}+\frac{1}{8 b^{2}}+\frac{1}{4 b^{3}}+\frac{1}{24 b^{4}} & \text { if } d=3 \\ \frac{11}{24 b^{2}}+\frac{1}{4 b^{3}}+\frac{1}{24 b^{4}} & \text { if } d=4 \\ \frac{1}{4 b}+\frac{11}{24 b^{2}}+\frac{1}{4 b^{3}}+\frac{1}{24 b^{4}} & \text { if } d \geq 5\end{cases}
$$

3.3. A weak lower bound for $t_{b, d}(m b)$. In this section we obtain an explicit but weak lower bound for $t_{b, d}(m b)$. We use this result in Lemma 3.3 to obtain a lower bound for the quantity $A(b, d, \varepsilon)$.

First we note that, if $d$ does not divide $m$, then $T_{b, d}(m b)$ contains all the $(m b)$ cycles and consequently,

$$
t_{b, d}(m b) \geq \frac{1}{m b} \quad \text { if } d \text { does not divide } m .
$$

We use this simple lower bound together with equation (5) to prove that a similar lower bound holds in all cases. In the proof and throughout the paper 'log' denotes the natural logarithm.

Lemma 3.1. Let $b, d, m$ be positive integers with $b, d \geq 2$. Then

$$
t_{b, d}(m b) \geq \begin{cases}\frac{b+\log m}{m b^{2}} & \text { if } m<d, \\ \frac{1}{m b} & \text { if d does not divide } m, \\ \frac{1}{m b^{2}} & \text { if d divides } m .\end{cases}
$$

In particular, $t_{b, d}(m b) \geq \frac{1}{m b^{2}}$.

Proof. By inequality (6), it remains to consider the cases where $m<d$ and where $d$ divides $m$. Suppose first that $m<d$. Then by equation (3) and inequality (6),

$$
m b t_{b, d}(m b)=\sum_{j=0}^{m-1} t_{b, d}(j b) \geq 1+\sum_{j=1}^{m-1} \frac{1}{j b}>1+\frac{1}{b} \int_{1}^{m} \frac{d x}{x}=\frac{b+\log m}{b}
$$

yielding $t_{b, d}(m b) \geq(b+\log m) /\left(m b^{2}\right)$, as asserted.

Next, if $m=d$, then by equation (4), and then (7),

$$
\begin{aligned}
d b t_{b, d}(d b) & =(d-1) b t_{b, d}((d-1) b)-1 \\
& >(d-1) b\left(\frac{b+\log (d-1)}{(d-1) b^{2}}\right)-1 \\
& =\frac{\log (d-1)}{b} .
\end{aligned}
$$

Thus $t_{b, d}(d b)>\frac{\log (d-1)}{d b^{2}}$ and this is at least the required lower bound $\frac{1}{d b^{2}}$ provided $\log (d-1) \geq 1$, that is, provided $d \geq 4$. The inequality $t_{b, d}(d b) \geq \frac{1}{d b^{2}}$ for $d=2,3$ follows from the exact values given in Section 3.2 .

Finally, we prove by induction that $t_{b, d}(\ell d b) \geq \frac{1}{\ell d b^{2}}$ for all $\ell \geq 1$. We have just proved this for $\ell=1$. Suppose that $\ell \geq 2$ and that $t_{b, d}((\ell-1) d b) \geq \frac{1}{(\ell-1) d b^{2}}$. Then by equation (5),

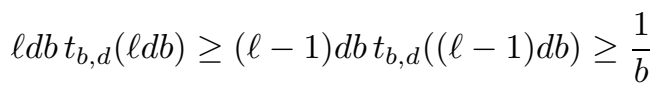

and the required lower bound follows by induction. 
3.4. The functions $A(b, d, \varepsilon)$ and $B(b, d)$. In this section we define the functions $A(b, d, \varepsilon)$ and $B(b, d)$ in Theorem 1.1 . We also prove simple upper and lower bounds for $A(b, d, \varepsilon)$ and $B(b, d)$.

Let $b, d$ be integers with $b, d \geq 2$. Set

$$
c(b, d)=\frac{1}{b}-\frac{1}{d b}=\frac{d-1}{d b}
$$

and

$$
f=f(b, d)=\frac{d-d^{c(b, d)}}{d-1}-\frac{1}{b} .
$$

Let $\varepsilon$ be a positive real number satisfying $\varepsilon \leq \frac{1}{2 d b}$. Define

$$
J(b, d, \varepsilon):=\left(\frac{(d-1)\left(1+\frac{1}{b}\right)}{\varepsilon d b}\right)^{\frac{d b}{(d-3 / 2)}} .
$$

Observe that $c(b, d)<1 / 2$, and $J(b, d, \varepsilon) \geq 4$ (see Lemma 3.2). We define

$$
A(b, d, \varepsilon):=\min _{1 \leq j \leq J(b, d, \varepsilon)}\left\{t_{b, d}(j b)(j b)^{1-c(b, d)+\varepsilon}\right\}
$$

and

$$
B(b, d):=\frac{1}{f(b, d) b^{c(b, d)}} .
$$

Lemma 3.2. Let $b, d$ be integers with $b, d \geq 2$, let $0<\varepsilon \leq \frac{1}{2 d b}$ and let $J(b, d, \varepsilon)$ be as defined in equation (10). Then

$$
d^{b} \leq J(b, d, \varepsilon)<1 /\left(4 \varepsilon^{1 / \varepsilon}\right) .
$$

Proof. Let $J=J(b, d, \varepsilon)$. Since $d \geq 2$, we have $\frac{d}{d-3 / 2} \leq 4$ and $(d-1) / d<1$. Therefore,

$$
J<\left(\frac{1+1 / b}{\varepsilon b}\right)^{4 b} .
$$

Since $(1+1 / b)^{b} \leq e$ for all $b \geq 2$, where $e$ is the base of the natural logarithm, we have

$$
J<\frac{e^{4}}{b^{4 b} \varepsilon^{4 b}} .
$$

Since $b \geq 2$, we have $b^{4 b} \geq 2^{8}$. Moreover, $e^{4} / 2^{8}<1 / 4$. Thus $J<1 /\left(4 \varepsilon^{4 b}\right)$. Since $\varepsilon \leq 1 /(2 d b)$ and $d \geq 2$, we have $4 b \leq 1 / \varepsilon$. Hence

$$
J<1 /\left(4 \varepsilon^{1 / \varepsilon}\right) .
$$

As $d \geq 2$, we have $d-1 \geq d / 2$. Moreover, $\frac{d b}{d-3 / 2}>b$. Therefore,

$$
J>\left(\frac{1}{2 \varepsilon b}\right)^{b} \geq d^{b}
$$

since $\varepsilon \leq 1 /(2 d b)$.

Now we compute upper and lower bounds for $A(b, d, \varepsilon)$ and $B(b, d)$. 
Lemma 3.3. Let $b, d$ be integers with $b, d \geq 2$ and $\varepsilon$ be a positive real number with $\varepsilon \leq 1 /(2 d b)$. Let $c=c(b, d)$ as defined in equation (8). Then

(i) $\frac{1}{b^{c}} \leq B(b, d) \leq \frac{12}{b^{c}}$.

(ii) $\frac{\varepsilon^{(c-\varepsilon) / \varepsilon}}{b^{1+c-\varepsilon}}<A(b, d, \varepsilon) \leq \frac{1}{b^{c-\varepsilon}}$.

Proof. (i) Observe that $\frac{d-\sqrt{d}}{d-1} \leq \frac{d-d^{c}}{d-1} \leq 1$ since $c \leq 1 / 2$. Since $\frac{d-\sqrt{d}}{d-1}$ is an increasing function for $d \geq 2$ we have $2-\sqrt{2} \leq \frac{d-d^{c}}{d-1} \leq 1$. Now, since $b \geq 2$, we have $1 / 12<3 / 2-\sqrt{2} \leq f(b, d) \leq 1$, (with $f(b, d)$ as in (9)) and the result follows.

(ii) Let $A=A(b, d, \varepsilon)$. By Lemma 3.1, $t_{b, d}(m b) \geq \frac{1}{m b^{2}}$ for all $m \geq 1$, so

$$
A \geq \min _{1 \leq j \leq J(b, d, \varepsilon)}\left\{j^{-c+\varepsilon} b^{-1-c+\varepsilon}\right\}
$$

and since $c>\varepsilon, j^{-c+\varepsilon}$ is decreasing as $j$ increases. Thus, by Lemma 3.2. $A \geq$ $\left(4 \varepsilon^{1 / \varepsilon}\right)^{c-\varepsilon} b^{-1-c+\varepsilon}$. Since $c-\varepsilon>0$, we have $A>\varepsilon^{(c-\varepsilon) / \varepsilon} b^{-1-c+\varepsilon}$. Moreover, since $J(b, d, \varepsilon) \geq 1$, we have $A \leq t_{b, d}(b) b^{1-c+\varepsilon}$ by the definition of $A$. As $t_{b, d}(b)=1 / b$, the result follows.

Remark 3.4. The first computer experimentation with the quantities $t_{b, d}(m b)$ was performed by Richard Crandall in December 2008. He was the first to observe 'sudden downspikes' in the values for $m$ a multiple of $d$. We made some further computer simulations to observe the growth of the function $g_{b, d, \varepsilon}(j)=t_{b, d}(j b)(j b)^{1-c(b, d)+\varepsilon}$. Representative results are given in Section 6. The results strongly suggest that the minimal value of $g_{b, d, \varepsilon}(j)$ occurs at $j=d$ and hence, by (11), $A(b, d, \varepsilon)$ is equal to $g_{b, d, \varepsilon}(d)$. If this observation were true for all integers $b, d \geq 2$ and all positive real numbers $\varepsilon \leq 1 /(2 d b)$, then by Lemma 3.1 , we would have

$$
A \geq \frac{(d b)^{1-c+\varepsilon}}{\left(d b^{2}\right)}=\frac{1}{b(d b)^{c-\varepsilon}}>\frac{1}{b^{1+c} d^{c}} .
$$

The bounds for $t_{b, d}(m b)$ presented in Theorem 1.1 would then become

$$
\frac{1}{b^{1+c} d^{c}} \frac{1}{(m b)^{1-\frac{1}{b}+\frac{1}{b d}+\varepsilon}} \leq t_{b, d}(m b) \leq \frac{12}{b^{c}} \frac{1}{(m b)^{1-\frac{1}{b}+\frac{1}{b d}}} .
$$

Since this lower bound would hold for any positive real number $\varepsilon \leq 1 /(2 d b)$, we could let $\varepsilon$ tend to 0 and obtain a lower bound

$$
t_{b, d}(m b) \geq \frac{1}{b^{1+c} d^{c}} \frac{1}{(m b)^{1-\frac{1}{b}+\frac{1}{b d}}}
$$

so that, indeed, $t_{b, d}(m b)=\Omega\left(m^{-1+\frac{1}{b}-\frac{1}{b d}}\right)$.

3.5. Proof of the upper bound in Theorem 1.1. We use the notation in Section 3.4. We need here and in the next subsection the following approximations; when $-1<a<0$ and $k \geq 1$,

$$
\int_{1}^{k} x^{a} d x \leq \sum_{l=1}^{k-1} l^{a} \leq 1+\int_{1}^{k-1} x^{a} d x
$$

and

$$
\text { (14) } \int_{0}^{\lceil k\rceil-1}(k-x)^{a} d x \leq \sum_{l=1}^{\lceil k\rceil-1}(k-l)^{a} \leq \int_{1}^{\lceil k\rceil-1}(k-x)^{a} d x+(k-\lceil k\rceil+1)^{a} \text {. }
$$


We fix $b, d \geq 2$ and set $t(m b)=t_{b, d}(m b)$ for simplicity. We prove by induction on $m$ that, for all $m \geq 1$,

$$
t(m b) \leq B(m b)^{-1+c},
$$

where $c=c(b, d)$ and $B=B(b, d)$ are as defined in equations (8) and (12), respectively.

By Lemma 3.3 (i), $B \geq b^{-c}$, so $t(b)=\frac{1}{b} \leq B b^{-1+c}$. Therefore, inequality (15) holds for $m=1$. Suppose inductively that $m>1$ and that inequality (15) holds for all $j<m$. We use equation (2) and apply the inductive hypothesis. Note that $m \in N_{d}(m)$ if and only if $d$ does not divide $m$, and that $t(0)=1$. Then equation (2) and inequalities (13) and (14) give

$$
\begin{aligned}
m b t(m b)= & \sum_{j \in N_{d}(m)} t((m-j) b) \\
\leq & 1+\sum_{j \in N_{d}(m), j<m} t((m-j) b) \\
\leq & 1+\sum_{j \in N_{d}(m), j<m} B((m-j) b)^{-1+c} \\
= & 1+B \sum_{\ell=1}^{m-1}(\ell b)^{-1+c}-B \sum_{\ell=1}^{\lceil m / d\rceil-1}((m-\ell d) b)^{-1+c} \\
\leq & 1+B b^{-1+c}\left(\int_{1}^{m-1} x^{-1+c} d x+1\right) \\
& -B(d b)^{-1+c} \int_{0}^{\lceil m / d\rceil-1}\left(\frac{m}{d}-x\right)^{-1+c} d x \\
= & 1+B b^{-1+c}\left(\left[\frac{x^{c}}{c}\right]_{1}^{m-1}+1\right)+B(d b)^{-1+c}\left[\frac{\left(\frac{m}{d}-x\right)^{c}}{c}\right]_{0}^{\lceil m / d\rceil-1} .
\end{aligned} .
$$

Using $m-1 \leq m, \frac{m}{d}-\left\lceil\frac{m}{d}\right\rceil+1 \leq 1$, we obtain

$$
m b t(m b) \leq 1+B b^{-1+c}\left(\frac{m^{c}}{c}-\frac{1}{c}+1\right)-B(d b)^{-1+c}\left(\frac{(m / d)^{c}}{c}-\frac{1}{c}\right),
$$

and since $\frac{1}{c}-\frac{1}{d c}=b$, the right-hand side equals

$$
1+B(m b)^{c}-B b^{-1+c}\left(\frac{1}{c}-1-\frac{d^{-1+c}}{c}\right) .
$$

We claim that $\frac{1}{c}-1-\frac{d^{-1+c}}{c} \geq \frac{b^{1-c}}{B}$. It follows from this inequality and the above upper bound that $t(m b) \leq B(m b)^{-1+c}$, and thus inequality (15) is proved by induction. It remains to prove the claim.

Substituting $c=\frac{d-1}{d b}$ where $c$ occurs as a denominator, and dividing by $b$, we see that the claimed inequality is equivalent to

$$
\frac{1}{B b^{c}} \leq \frac{d}{d-1}-\frac{1}{b}-\frac{d^{c}}{d-1}=\frac{d-d^{c}}{d-1}-\frac{1}{b}=f(b, d),
$$

and the truth of this inequality follows from the definition of $B$ in equation (12). This completes the proof of inequality (15). 
3.6. Proof of the lower bound in Theorem 1.1. We again use the notation in Section 3.4. We keep $b, d$ fixed, where $b, d \geq 2$, and set $t(m b)=t_{b, d}(m b)$.

We prove by induction on $m$ that, for all $m \geq 1$,

$$
t(m b) \geq \frac{A}{(m b)^{1-\frac{1}{b}+\frac{1}{d b}+\varepsilon}}=A(m b)^{-1+c-\varepsilon}
$$

where $c=c(b, d)$ and $A=A(b, d, \varepsilon)$ are as defined in equations (8) and (11), respectively, and $0<\varepsilon \leq 1 / 2 d b$. Note that $1-c+\varepsilon>1 / 2+\varepsilon>0$. By the definition of $A$, inequality (16) holds if $1 \leq m \leq J(b, d, \varepsilon)$. Suppose that $m>J(b, d, \varepsilon)$ and that equation (16) holds for all $j \leq m-1$. Then, by equation (2) and inequalities (13) and (14),

$$
\begin{aligned}
m b t(m b)= & \sum_{j \in N_{d}(m)} t((m-j) b) \geq \sum_{j \in N_{d}(m) \backslash\{m\}} A((m-j) b)^{-1+c-\varepsilon} \\
= & A \sum_{\ell=1}^{m-1}(\ell b)^{-1+c-\varepsilon}-A \sum_{\ell=1}^{\left\lceil\frac{m}{d}\right\rceil-1}((m-\ell d) b)^{-1+c-\varepsilon} \\
\geq & A b^{-1+c-\varepsilon} \int_{1}^{m} x^{-1+c-\varepsilon} d x \\
& -A(d b)^{-1+c-\varepsilon} \int_{1}^{\left\lceil\frac{m}{d}\right\rceil-1}\left(\frac{m}{d}-x\right)^{-1+c-\varepsilon} d x \\
& -A(d b)^{-1+c-\varepsilon}\left(\frac{m}{d}-\left\lceil\frac{m}{d}\right\rceil+1\right)^{-1+c-\varepsilon} \\
= & \frac{A}{b^{1-c+\varepsilon}}\left(\left\lceil\frac{x^{c-\varepsilon}}{c-\varepsilon}\right]_{1}^{m}+\frac{1}{d^{1-c+\varepsilon}}\left[\frac{\left(\frac{m}{d}-x\right)^{c-\varepsilon}}{c-\varepsilon}\right]_{1}^{\left\lceil\frac{m}{d}\right\rceil-1}\right) \\
& -\frac{A}{(b d)^{1-c+\varepsilon}}\left(\frac{m}{d}-\left\lceil\frac{m}{d}\right\rceil+1\right)^{-1+c-\varepsilon} \cdot
\end{aligned}
$$

Using $\frac{m}{d}-\left\lceil\frac{m}{d}\right\rceil+1 \geq \frac{1}{d}$ and $\left(\frac{m}{d}-1\right) \leq \frac{m}{d}$, we have

$$
\begin{aligned}
m b t(m b) \geq & \frac{A}{b^{1-c+\varepsilon}} \frac{\left(m^{c-\varepsilon}-1\right)}{c-\varepsilon}+\frac{A}{d(b)^{1-c+\varepsilon}} \frac{1}{c-\varepsilon} \\
& -\frac{A}{(d b)^{1-c+\varepsilon}} \frac{\left(\frac{m}{d}\right)^{c-\varepsilon}}{c-\varepsilon}-\frac{A}{b^{1-c+\varepsilon}} .
\end{aligned}
$$

After rearranging the terms, we get

$$
m b t(m b) \geq \frac{A}{b^{1-c+\varepsilon}} \frac{m^{c-\varepsilon}}{c-\varepsilon}\left(1-\frac{1}{d}\right)-\left(1-\frac{1}{d}\right) \frac{A}{b^{1-c+\varepsilon}} \frac{1}{c-\varepsilon}-\frac{A}{b^{1-c+\varepsilon}} .
$$

Now, we claim that

$$
\frac{A}{b^{1-c+\varepsilon}} \frac{m^{c-\varepsilon}}{c-\varepsilon}\left(1-\frac{1}{d}\right)-\left(1-\frac{1}{d}\right) \frac{A}{b^{1-c+\varepsilon}} \frac{1}{c-\varepsilon}-\frac{A}{b^{1-c+\varepsilon}} \geq A(m b)^{c-\varepsilon}
$$

or, equivalently, multiplying both sides by $\frac{d b^{1-c+\varepsilon}(c-\varepsilon)}{A m^{c-\varepsilon}}$,

$$
(d-1)-\frac{d-1}{m^{c-\varepsilon}}-\frac{d(c-\varepsilon)}{m^{c-\varepsilon}} \geq d b(c-\varepsilon) .
$$


It follows from inequality (17) and the proof of inequality (18) that $t(m b) \geq$ $A(b, d, \varepsilon)(m b)^{-1+c-\varepsilon}$, and thus inequality (16) is proved by induction. It remains to prove inequality (18).

By our assumptions $m>J(b, d, \varepsilon)$, and so, by raising both sides of this inequality to the power $\frac{d-3 / 2}{b d}=\frac{1}{b}-\frac{3 / 2}{d b}$, and using (10), we have

$$
m^{\frac{1}{b}-\frac{3 / 2}{d b}} \geq \frac{d-1}{\varepsilon d b}\left(1+\frac{1}{b}\right)
$$

Hence

$$
\varepsilon d b \geq \frac{1}{m^{\frac{1}{b}-\frac{3 / 2}{d b}}}\left(d-1+\frac{d-1}{b}\right) .
$$

Since $\varepsilon \leq \frac{1}{2 d b}$, we have $\frac{1}{b}-\frac{1}{d b}-\varepsilon \geq \frac{1}{b}-\frac{3}{2 d b}$, and hence

$$
\varepsilon d b \geq \frac{1}{m^{\frac{1}{b}-\frac{1}{d b}-\varepsilon}}\left(d-1+\frac{d-1-\varepsilon d b}{b}\right) .
$$

Recall that $c=\frac{1}{b}-\frac{1}{d b}=\frac{d-1}{d b}$ so $d-1-\varepsilon b d=b d(c-\varepsilon)$ and $\varepsilon b d=d-1-b d(c-\varepsilon)$. Thus, substituting for these values in (19) and rearranging, we get

$$
d-1-\frac{d-1}{m^{c-\varepsilon}}-\frac{d(c-\varepsilon)}{m^{c-\varepsilon}} \geq b d(c-\varepsilon)
$$

and the claim is proved.

3.7. Proof of the corollaries. In this section we prove Corollaries 1.2 and 1.4 .

Proof of Corollary 1.2. It follows from the definition of a pre-semiregular permutation that the proportion of the pre-semiregular elements of order $p^{k}$ on a set of size $p^{k} m$ is at least $t_{p^{k}, p}\left(p^{k} m\right)$, and hence the result follows from Theorem 1.1.

Proof of Corollary 1.4. Let $d$ be an integer, $d \geq 4$. Suppose that $n$ has a nontrivial divisor which is at most $d$. Then there is a prime $p$ dividing $n$ with $p \leq d$. We apply Corollary 1.2 with $k=1$ and $\varepsilon=1 /\left(2 p^{2}\right)$. Note that $1-\frac{p-1}{p^{k+1}}+\varepsilon=1-\frac{1}{p}+\frac{3}{2 p^{2}}$ and this is an increasing function of $p$ for $p \geq 3$, so if $p$ is odd, this is at most $1-\frac{1}{d}+\frac{3}{2 d^{2}}<1-\frac{1}{2 d}$ since $d \geq 4$. If $p=2$, then $1-\frac{1}{p}+\frac{3}{2 p^{2}}=7 / 8$ and since $d \geq 4$, we have $1-1 /(2 d) \geq 7 / 8$. Thus, by Corollary 1.2 , the proportion of pre-semiregular elements of order $p$ is at least $A\left(p, p, \frac{1}{2 p^{2}}\right) n^{-1+\frac{1}{2 d}}$. By defining $C(d)$ as the minimum of $A\left(p, p, \frac{1}{2 p^{2}}\right)$ over all prime divisors $p$ of $n$ such that $p \leq d$, the result follows.

\section{A generating Function For The $t_{b, d}(m b)$}

In this section we prove Theorem 1.5. Recall that

$$
T(x)=\sum_{m \geq 0} t_{b, d}(m b) x^{m}
$$

and $t_{b, d}(0)=1$.

Proof of Theorem 1.5. We have $T^{\prime}(x)=\sum_{m>1} m t_{b, d}(m b) x^{m-1}$. Write $T^{\prime}(x)=$ $F_{1}(x)+F_{2}(x)$ where $F_{1}(x)=\sum_{m=1}^{d} m t_{b, d}(m b) x^{m-1}$ and $F_{2}(x)=T^{\prime}(x)-F_{1}(x)$. 
For $|x|<1$, the recursion in equation (5) yields the following expression for $F_{2}(x)$ :

$$
\begin{aligned}
F_{2}(x)= & \frac{1}{b} \sum_{m \geq d+1}\left((m-d) b t_{b, d}((m-d) b)+\sum_{j=1}^{d-1} t_{b, d}((m-j) b)\right) x^{m-1} \\
= & x^{d} \sum_{m-d \geq 1}(m-d) t_{b, d}((m-d) b) x^{m-d-1} \\
& +\frac{1}{b} \sum_{j=1}^{d-1} x^{j-1}\left(\sum_{m-j \geq d-j+1} t_{b, d}((m-j) b) x^{m-j}\right) \\
= & x^{d} T^{\prime}(x)+\frac{1}{b} \sum_{j=1}^{d-1} x^{j-1}\left(T(x)-\sum_{k=0}^{d-j} t_{b, d}(k b) x^{k}\right) \\
= & x^{d} T^{\prime}(x)+\frac{T(x)}{b} \cdot \frac{1-x^{d-1}}{1-x}-F_{3}(x)
\end{aligned}
$$

where

$$
F_{3}(x)=\frac{1}{b} \sum_{j=1}^{d-1}\left(\sum_{k=0}^{d-j} t_{b, d}(k b) x^{j+k-1}\right) .
$$

We obtain a different expression for $F_{3}(x)$. For $0 \leq m \leq d-1$, the coefficient of $x^{m}$ in $F_{3}(x)$ is $\frac{1}{b} \sum_{j=1}^{\min \{m+1, d-1\}} t_{b, d}((m-j+1) b)$. If $m=d-1$ this is $\sum_{\ell=1}^{d-1} t_{b, d}(\ell b)$ while if $0 \leq m \leq d-2$ this is $\frac{1}{b} \sum_{j=0}^{m} t_{b, d}(j b)$. Thus

$$
F_{3}(x)=\frac{1}{b}\left(\sum_{m=0}^{d-2}\left(\sum_{\ell=0}^{m} t_{b, d}(\ell b)\right) x^{m}+\sum_{\ell=1}^{d-1} t_{b, d}(\ell b) x^{d-1}\right)
$$

Also, using equations (3) and (4) we obtain a new expression for $F_{1}(x)$ :

$$
\begin{aligned}
F_{1}(x) & =\sum_{m=1}^{d} m t_{b, d}(m b) x^{m-1} \\
& =\sum_{m=1}^{d-1}\left(\frac{1}{b} \sum_{j=0}^{m-1} t_{b, d}(j b)\right) x^{m-1}+\frac{1}{b} \sum_{j=1}^{d-1} t_{b, d}(j b) x^{d-1} \\
& =\frac{1}{b}\left(\sum_{m=1}^{d-1}\left(\sum_{j=0}^{m-1} t_{b, d}(j b)\right) x^{m-1}+\sum_{j=1}^{d-1} t_{b, d}(j b) x^{d-1}\right) \\
& =F_{3}(x) .
\end{aligned}
$$

Hence

$$
T^{\prime}(x)=F_{1}(x)+F_{2}(x)=x^{d} T^{\prime}(x)+\frac{T(x)}{b} \cdot \frac{1-x^{d-1}}{1-x},
$$

which implies that, for $|x|<1$,

$$
\frac{T^{\prime}(x)}{T(x)}=\frac{1-x^{d-1}}{b(1-x)} \cdot \frac{1}{1-x^{d}}=\frac{1}{b}\left(\frac{1}{1-x}-\frac{x^{d-1}}{1-x^{d}}\right) .
$$


Integrating both sides, we obtain

$$
\begin{aligned}
\log (T(x)) & =\frac{1}{b}\left(\int \frac{d x}{1-x}-\int \frac{x^{d-1} d x}{1-x^{d}}\right) \\
& =\frac{1}{b}\left(-\log (1-x)+\frac{1}{d} \log \left(1-x^{d}\right)\right)+c \\
& =\log \left(\frac{\left(1-x^{d}\right)^{1 / b d}}{(1-x)^{1 / b}}\right)+c
\end{aligned}
$$

and as $T(0)=t_{b, d}(0)=1$ we have $c=0$. Thus

$$
T(x)=\frac{\left(1-x^{d}\right)^{1 / b d}}{(1-x)^{1 / b}} .
$$

\section{AN EXPRESSION FOR THE $t_{b, d}(m b)$}

In this section we derive the following expression for the $t_{b, d}(m b)$.

Proposition 5.1. Let $m, b, d$ be integers with $b, d \geq 2$ and $m \geq 1$. Then

$$
t_{b, d}(m b)=s_{\neg \hat{b}}(m \hat{b})-\frac{1}{d b} \sum_{k=1}^{\left\lfloor\frac{m}{d}\right\rfloor} \frac{1}{k} s_{\neg \hat{b}}((m-d k) \hat{b}) s_{\neg d b}(d b k-1)
$$

where $\hat{b}=\frac{b}{b-1}$, and for positive real numbers $q, t$ with $q>1, s_{\neg q}(0)=1$ and

$$
s_{\neg q}(t)=\prod_{i=1}^{\lfloor t / q\rfloor}\left(1-\frac{1}{q i}\right) .
$$

If $q$ is an integer, $q \geq 2$, then the quantity $s_{\neg q}(n)$ has a combinatorial significance for positive integers $n$, namely $s_{\neg q}(n)$ is the proportion of elements in $S_{n}$ with no cycle lengths divisible by $q$ (see for example [1, Theorem 2.3(a)]), an easy extension of the proof in [5] for prime powers $q$. It was proved in [1, Theorem 2.3(b)] that $s_{\neg q}(n)=\Theta\left(n^{-1 / q}\right)$ for an integer $q \geq 2$. The same proof shows that $s_{\neg q}(n q)=$ $\Theta\left(n^{-1 / q}\right)$ for a real number $q>1$.

For an integer $k \geq 2$, let $S_{\neg k}(x)$ be the ordinary generating function for $s_{\neg k}(n)$, that is,

$$
S_{\neg k}(x)=\sum_{n \geq 0} s_{\neg k}(n) x^{n} .
$$

By the proof of Theorem 2.1 in [14, for $|x|<1$, we have

$$
S_{\neg k}(x)=\frac{\left(1-x^{k}\right)^{1 / k}}{1-x} .
$$

Lemma 5.2. Let $b, d$ be integers with $b, d \geq 2$ and let $T(x)$ be as in equation (20). Then, for $|x|<1$,

$$
T\left(x^{b}\right)=\frac{S_{\neg d b}(x)}{S_{\neg b}(x)} .
$$

Proof. This follows immediately from (22) and Theorem 1.5 by substituting $x^{b}$ for $x$ in $T(x)$. 
For a real number $q>1$, let $s_{\neg q}(0)=1$ and let $\hat{S}_{\neg q}(x)$ be the generating function

$$
\hat{S}_{\neg q}(x)=\sum_{m \geq 0} s_{\neg q}(q m) x^{m} .
$$

It turns out that the reciprocal $1 / S_{\neg k}(x)$, for $k$ a positive integer, is related to $\hat{S}_{\neg q}(x)$ with $q=\frac{k}{k-1}=\hat{k}$.

Lemma 5.3. Let $k$ be an integer with $k \geq 2$, and set $\hat{k}=k /(k-1)$. Then, for $|x|<1$, we get:

(i) $\hat{S}_{\neg \hat{k}}(x)=(1-x)^{-1 / k}$.

(ii) $\frac{1}{S_{\neg k}(x)}=(1-x) \hat{S}_{\neg \hat{k}}\left(x^{k}\right)$.

Proof. (i) For $|x|<1$, by (22),

$$
\frac{1}{S_{\neg k}(x)}=(1-x)\left(1-x^{k}\right)^{-1 / k} .
$$

Consider the power series expansion $(1-x)^{-1 / k}=\sum_{m=0}^{\infty} g(k, m) x^{m}$. Evaluating at $x=0$ yields $g(k, 0)=1=s_{\neg \hat{k}}(0)$. Using a 'binomial type' expansion, we have, for $m \geq 1$,

$$
\begin{aligned}
g(k, m) & =(-1)^{m} \frac{1}{m !}\left(-\frac{1}{k}\right)\left(-\frac{1}{k}-1\right)\left(-\frac{1}{k}-2\right) \cdots\left(-\frac{1}{k}-m+1\right) \\
& =\frac{1}{m !} \cdot \frac{1}{k} \cdot\left(\frac{1}{k}+1\right) \cdots\left(\frac{1}{k}+m-1\right) \\
& =\frac{1}{m k} \prod_{i=1}^{m-1}\left(1+\frac{1}{i k}\right) .
\end{aligned}
$$

In particular, $g(k, 1)=\frac{1}{k}=s_{\neg \hat{k}}(\hat{k})$. This yields a recursion for the $g(k, m)$, namely, for all $m \geq 2$,

$$
\begin{aligned}
g(k, m) & =\frac{1}{m k} \prod_{i=1}^{m-1}\left(1+\frac{1}{i k}\right) \\
& =\frac{m-1}{m} g(k, m-1)\left(1+\frac{1}{(m-1) k}\right) \\
& =g(k, m-1)\left(1-\frac{k-1}{m k}\right) \\
& =g(k, m-1)\left(1-\frac{1}{m \hat{k}}\right) .
\end{aligned}
$$

We note that this relation also holds for $m=1$, that is, $g(k, 1)=\frac{1}{k}=g(k, 0)\left(1-\frac{1}{\hat{k}}\right)$. An easy inductive proof gives, for $m \geq 1$, that

$$
g(k, m)=\prod_{j=1}^{m}\left(1-\frac{1}{\hat{k} j}\right)=s_{\neg \hat{k}}(\hat{k} m) .
$$

Thus $(1-x)^{-1 / k}=\hat{S}_{\neg \hat{k}}(x)$ proving part (i). Part (ii) follows immediately from (i) in view of equation (22) for $S_{\neg k}(x)$. 
Proof of Proposition [5.1. Combining Corollary [5.2 and Lemma 5.3, we have

$$
\begin{aligned}
T\left(x^{b}\right)= & \frac{S_{\neg d b}(x)}{S_{\neg b}(x)} \\
= & \left(\sum_{\ell=0} s_{\neg \hat{b}}(\ell \hat{b}) x^{\ell b}\right)(1-x)\left(\sum_{n=0} s_{\neg d b}(n) x^{n}\right) \\
= & \left(\sum_{\ell=0} s_{\neg \hat{b}}(\ell \hat{b}) x^{\ell b}\right)\left(\sum_{n=0} s_{\neg d b}(n) x^{n}\right) \\
& -\left(\sum_{\ell=0} s_{\neg \hat{b}}(\ell \hat{b}) x^{\ell b}\right)\left(\sum_{n=0} s_{\neg d b}(n) x^{n+1}\right) .
\end{aligned}
$$

Thus the coefficient $t_{b, d}(m b)$ of $x^{m b}$ in $T\left(x^{b}\right)$ is equal to

$$
t_{b, d}(m b)=\sum_{\ell=0}^{m} s_{\neg \hat{b}}(\ell \hat{b}) s_{\neg d b}(m b-\ell b)-\sum_{\ell=0}^{m-1} s_{\neg \hat{b}}(\ell \hat{b}) s_{\neg d b}(m b-\ell b-1) .
$$

Separating out the term for $\ell=m$ in the first summation, we get

$$
t_{b, d}(m b)=s_{\neg \hat{b}}(m \hat{b})+\sum_{\ell=0}^{m-1} s_{\neg \hat{b}}(\ell \hat{b})\left(s_{\neg d b}(m b-\ell b)-s_{\neg d b}(m b-\ell b-1)\right) .
$$

If $m b-\ell b=(m-\ell) b$ is not a multiple of $d b$, then $m-\ell$ is not a multiple of $d$ and $s_{\neg d b}(m b-\ell b)=s_{\neg d b}(m b-\ell b-1)$ by [1, Lemma 2.2]. Therefore, the only values of $\ell$ for which there is a nonzero contribution in the summation are those of the form $\ell=m-d k$ for some integer $k$ such that $1 \leq k \leq\left\lfloor\frac{m}{d}\right\rfloor$. Then $m=\ell+d k$, and equation (24) becomes

$$
t_{b, d}(m b)=s_{\neg \hat{b}}(m \hat{b})+\sum_{k=1}^{\left\lfloor\frac{m}{d}\right\rfloor} s_{\neg \hat{b}}((m-d k) \hat{b})\left(s_{\neg d b}(d b k)-s_{\neg d b}(d b k-1)\right) .
$$

It follows from equation (21) that $s_{\neg d b}(d b k)-s_{\neg d b}(d b k-1)=-\frac{1}{d b k} s_{\neg d b}(d b k-1)$. Hence we have

$$
t_{b, d}(m b)=s_{\neg \hat{b}}(m \hat{b})-\frac{1}{d b} \sum_{k=1}^{\left\lfloor\frac{m}{d}\right\rfloor} \frac{1}{k} s_{\neg \hat{b}}((m-d k) \hat{b}) s_{\neg d b}(d b k-1) .
$$

6. Computer simulations to estimate $A(b, d, \varepsilon)$

Recall that

$$
A(b, d, \varepsilon)=\min _{1 \leq j \leq J(b, d, \varepsilon)} g_{b, d, \varepsilon}(j),
$$

where $g_{b, d, \varepsilon}(j)=t_{b, d}(j b)(j b)^{1-c(b, d)+\varepsilon}$ and $J(b, d, \varepsilon)$ is given by (10).

We present some computer simulations which suggest that, in fact,

$$
A(b, d, \varepsilon)=g_{b, d, \varepsilon}(d),
$$

as noted in Remark 3.4. In Figures 1, 2 and 3, we give three examples, one for each of $d=2,3,4$. In each case we present two plots, one showing only the first 
few values of $j$, and another showing a larger range of $j$. Note that it is impractical to plot over the entire range $1 \leq j \leq J(b, d, \varepsilon)$, since $J(b, d, \varepsilon)$ can be extremely large and calculation of the $t_{b, d}(j b)$ (via Proposition 5.1) becomes computationally intensive for large $j$.
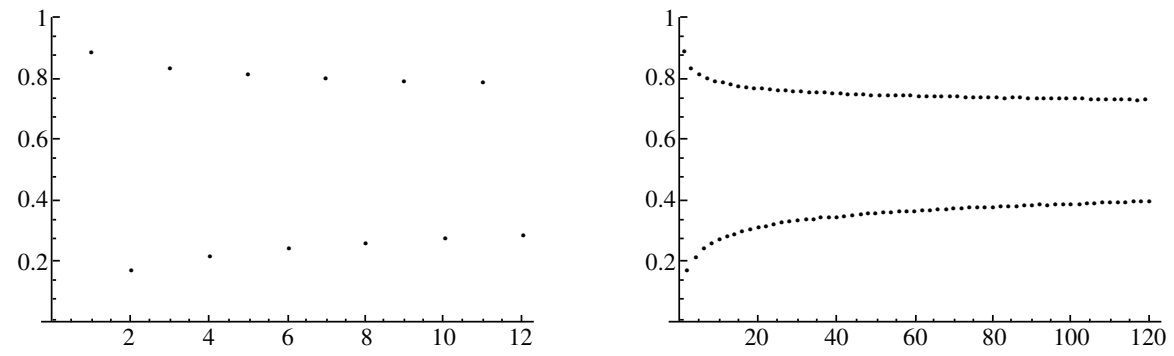

Figure 1. Plot of $g_{b, d, \varepsilon}(j)$ with $b=5, d=2$ and $\varepsilon=1 / 40<$ $1 /(2 d b)$, for $1 \leq j \leq 12$ and $1 \leq j \leq 120<J(b, d, \varepsilon) \approx 4.2 \times 10^{13}$.
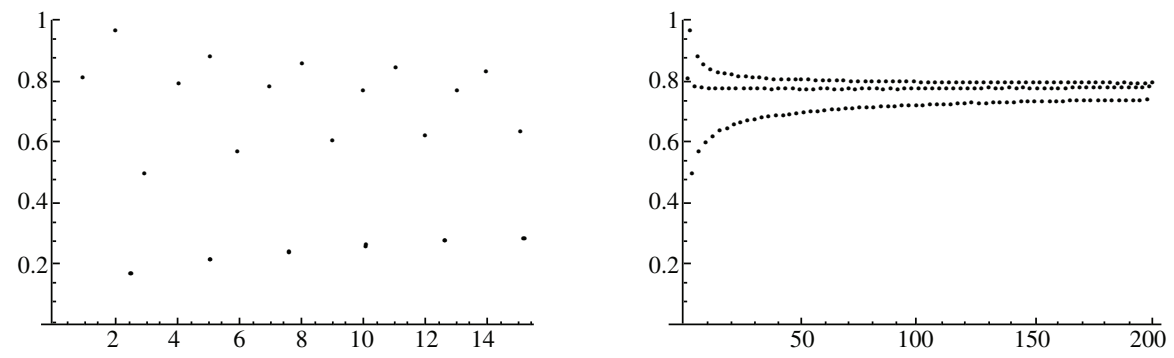

Figure 2. Plot of $g_{b, d, \varepsilon}(j)$ with $b=2, d=3$ and $\varepsilon=1 / 80<$ $1 /(2 d b)$, for $1 \leq j \leq 14$ and $1 \leq j \leq 200<J(b, d, \varepsilon) \approx 2.5 \times 10^{6}$.
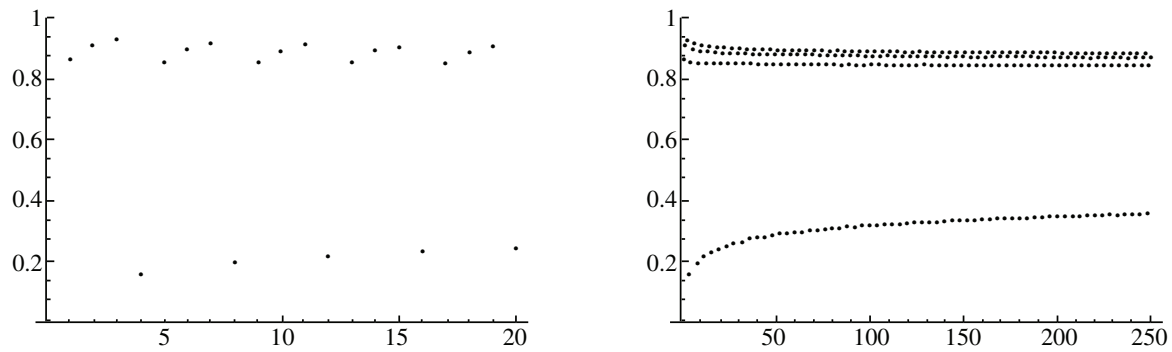

Figure 3. Plot of $g_{b, d, \varepsilon}(j)$ with $b=10, d=4$ and $\varepsilon=1 / 100<$ $1 /(2 d b)$, for $1 \leq j \leq 20$ and $1 \leq j \leq 250<J(b, d, \varepsilon) \approx 4.6 \times 10^{14}$. 
The plots - which seem to be representative (based on several other examples which we have computed) - suggest that

(i) the value of $g_{b, d, \varepsilon}(j)$ depends on the congruence class of $j$ modulo $d$, in the sense that each class corresponds to one of $d$ 'curves';

(ii) the lowest 'curve' in each plot corresponds to those $j$ values which are multiples of $d$, and $g_{b, d, \varepsilon}(\ell d)$ is strictly increasing in $\ell$;

(iii) the remaining 'curve' are bounded away from the lowest one (and, for fixed $k \in\{1, \ldots, d-1\}, g_{b, d, \varepsilon}(\ell d+k)$ is strictly decreasing in $\left.\ell\right)$.

In particular, the plots seem to confirm that $g_{b, d, \varepsilon}(j)$ attains its minimum value when $j=d$.

\section{ACKNOWLEDGEMENTS}

The authors are indebted first to John Borwein and Richard Crandall (see Remark (3.4). They also thank Peter Neumann and Lyle Noakes for helpful discussions and an anonymous referee for a careful report on the paper. The results of this paper form part of ARC Federation Fellowship project FF0776186 and Discovery project DP0879134.

\section{REFERENCES}

[1] R. Beals, C. R. Leedham-Green, A. C. Niemeyer, C. E. Praeger, and Á. Seress. Permutations with restricted cycle structure and an algorithmic application. Combin. Probab. Comput., 11(5):447-464, 2002. MR1930351 (2003h:60012)

[2] P. Cameron (ed.). Problems from the fifteenth british combinatorial conference. Discrete Math., (167/168):605-615, 1997.

[3] P. J. Cameron, M. Giudici, G. A. Jones, W. M. Kantor, M. H. Klin, D. Marušič, and L. A. Nowitz. Transitive permutation groups without semiregular subgroups. J. London Math. Soc. (2), 66(2):325-333, 2002. MR.1920405 (2003f:20001)

[4] P. Cameron, J. Sheehan, and P. Spiga. Semiregular automorphisms of vertex-transitive cubic graphs. European J. Combin., 27(6):924-930, 2006. MR2226427(2006m:05107)

[5] P. Erdős and P. Turán. On some problems of a statistical group-theory. II. Acta math. Acad. Sci. Hungar., 18:151-163, 1967. MR0207810 (34:7624)

[6] B. Fein, W. M. Kantor, and M. Schacher. Relative Brauer groups. II. J. Reine Angew. Math., 328:39-57, 1981. MR636194 (83a:12018)

[7] M. Giudici. Quasiprimitive groups with no fixed point free elements of prime order. J. London Math. Soc. (2), 67(1):73-84, 2003. MR1942412 (2003m:20001)

[8] M. Giudici and S. Kelly. Characterizing a family of elusive permutation groups. J. Group Theory, 12(1):95-105, 2009. MR2488141 (2010a:20002)

[9] M. Giudici and J. Xu. All vertex-transitive locally-quasiprimitive graphs have a semiregular automorphism. J. Alg. Combin., 25(2):217-232, 2007. MR2310422(2008g:05093)

[10] Seok-Hee Hong, Peter Eades, Aaron Quigley and Sang-Ho Lee. Drawing algorithms for seriesparallel digraphs in two and three dimensions, in Graph drawing: 6th international symposium, GD '98, Montreal Canada, August 1998. Proceedings, Ed: Sue H. Whitesides, Lecture Notes in Computer Science 1547, Springer, Berlin, 1998. MR1717452

[11] D. Jordan. Eine Symmetrieeigenschaft von Graphen, Graphentheorie und ihre Anwendungen (Stadt Wehlen, 1988). Dresdner Reihe Forsch. 9, pages 17-20, 1988. MR979101 (90c:05106)

[12] Cai Heng Li. Semiregular automorphisms of cubic vertex transitive graphs. Proc. Amer. Math. Soc., 136(6):1905-1910, 2008. MR2383495 (2008m:05144)

[13] Brendan McKay and Gordon F. Royle, The transitive graphs with at most 26 vertices. Ars Combin, 30:161-176, 1990. MR:1088288 (91j:05056)

[14] Attila Maróti. Symmetric functions, generalized blocks, and permutations with restricted cycle structure. European J. Combin., 28(3):942-963, 2007. MR2300774(2009b:05009)

[15] D. Marušič. On vertex symmetric digraphs. Discrete Math., 36(1):69-81, 1981. MR621894 (83a:05068) 
[16] D. Marušič and R. Scapellato. Permutation groups, vertex-transitive digraphs and semiregular automorphisms. European J. Combin., 19(6):707-712, 1998. MR.1642726 (99g:05101)

[17] P.-R. de Montfort, Essai d'Analyse sur les Jeux de Hasard Paris: Jacque Quillau, 1708. Second Edition, Revue et augmentée de plusieurs Lettres. Paris: Jacque Quillau. 1713.

School of Mathematics and Statistics, The University of Western Australia, Perth, WA, 6009, Australia

E-mail address: Alice.Niemeyer@uwa.edu.au

School of Mathematics and Statistics, The University of Western Australia, Perth, WA, 6009, Australia

E-mail address: Tomasz.Popiel@uwa.edu.au

School of Mathematics and Statistics, The University of Western Australia, Perth, WA, 6009, Australia

E-mail address: Cheryl.Praeger@uwa.edu.au

School of Mathematics and Statistics, The University of Western Australia, Perth, WA, 6009, Australia

E-mail address: sukru@maths.uwa.edu.au 